\title{
Textile-based triboelectric nanogenerator with alternating positive and negative freestanding woven structure for harvesting sliding energy in all directions
}

\author{
Watcharapong Paosangthonga,*, Mahmoud Wagiha, Russel Torah ${ }^{a}$, Steve Beeby \\ ${ }^{a}$ School of Electronics and Computer Science, University of Southampton, Southampton, SO171BJ, UK \\ *Corresponding author \\ E-mail addresses:wp1y15@soton.ac.uk (W.Paosangthong),_mahm1g15@soton.ac.uk (M.Wagih),rnt@ecs.soton.ac.uk (R. \\ Torah),spb@ecs.soton.ac.uk (S.Beeby)
}

\begin{abstract}
A novel textile-based triboelectric nanogenerator (TENG) with woven structure operating in freestanding triboelectric-layer mode, defined as a woven-TENG, has been developed. Whereas most woven-structured TENGs operate in contact-separation mode and consist of one type of triboelectric material, this woven-TENG comprises woven electrodes and woven strips of positive and negative triboelectric material, which form a checker-like pattern over the electrodes with matching periodicity. The implementation of the positive and negative triboelectric material significantly improves the performance of the woven-TENG. Furthermore, in contrast to the conventional grating-structured and woven-structured TENG, which are designed to operate only in one moving direction, this new design also allows the woven-TENG to harvest energy from allplanar directions of movement. The woven-TENG with woven strips of nylon and polytetrafluoroethylenevinyl (PTFE) fabric can generate a root mean square (RMS) open-circuit voltage of $62.9 \mathrm{~V}$, an RMS short-circuit current of $1.77 \mu \mathrm{A}$ and a maximum RMS power of 34.8 $\mu \mathrm{W}$ at a load resistance of $50 \mathrm{M} \Omega$, a mechanical oscillation of $2 \mathrm{~Hz}$ and a contact force of $5 \mathrm{~N}$. This corresponds to a maximum RMS power density of $5.43 \mathrm{~mW} / \mathrm{m}^{2}$.
\end{abstract}

KEYWORDS: Triboelectric nanogenerator, e-textile, woven structure, all directions harvester 


\section{Introduction}

Recently, wearable and portable electronics are penetrating into our daily life. They are more sophisticated and multifunctional but extensively rely on batteries, which demand frequent recharging and replacement. To overcome this limitation, self-powered wearable systems using a biomechanical energy harvester have been intensively researched and triboelectric nanogenerators (TENGs) have become one of the most promising choices. Since the first discovery in 2012 [1], significant advances have been made both in their performance [2-7] and versatility [8-10]. Due to their properties, including low weight, flexibility, washability and biocompatibility, they are highly suitable for wearable applications. As a result, textile-based TENGs have attracted particular interest and numerous examples have been demonstrated [11-15].

Most TENGs operating in freestanding triboelectric-layer mode (FT-mode) can only harvest energy in one translational direction $[16,17]$ or rotational direction $[18,19]$. The first TENG, which can generate energy from all sliding directions has been proposed by Guo et al [20]. They reported a checker-like TENG with interdigitated electrodes and used a sliding contact between PET and PTFE as a source for the triboelectric charge generation. Next, Xia et al. has redesigned the electrodes by introducing a new honeycomb-like three-electrodes structure [21]. Recently, a similar idea to the checker-like TENG has been proposed by Ma et al. They developed a biomimetic fish-scale-like TENG and manufactured based on PET film and PTFE sheet [22]. However, these examples are made of rigid materials, which are inappropriate for wearable applications and the latter also suffers from a lower effective contact area, since only a small part of the top substrate can make a contact with the bottom substrate. Additionally, Chen et al. have manually knitted a mosaic patterned TENG for all-direction sliding energy harvesting [23]. However, this work did not demonstrate its successful operation as an energy harvester.

This paper proposed a novel textile-based TENG with woven structure (woven-TENG). While most woven-structured TENGs operate in contact-separation mode and consist of one type of triboelectric material [24-27], this woven-TENG comprises woven electrodes and woven strips of positive and negative triboelectric material and operates in the sliding FT-mode. Because of the nature of the woven structure, a symmetric and periodic arrangement of the electrodes and alternating triboelectric materials are self-formed. The implementation of the alternating positive and negative triboelectric material can considerably enhance the performance of a TENG operating 
in FT-mode [28]. In addition, the fabrication processes of the woven-TENG, including doctorblading and weaving, are common, low-cost and compatible with standard textile manufacturing.

\section{Design and methodology}
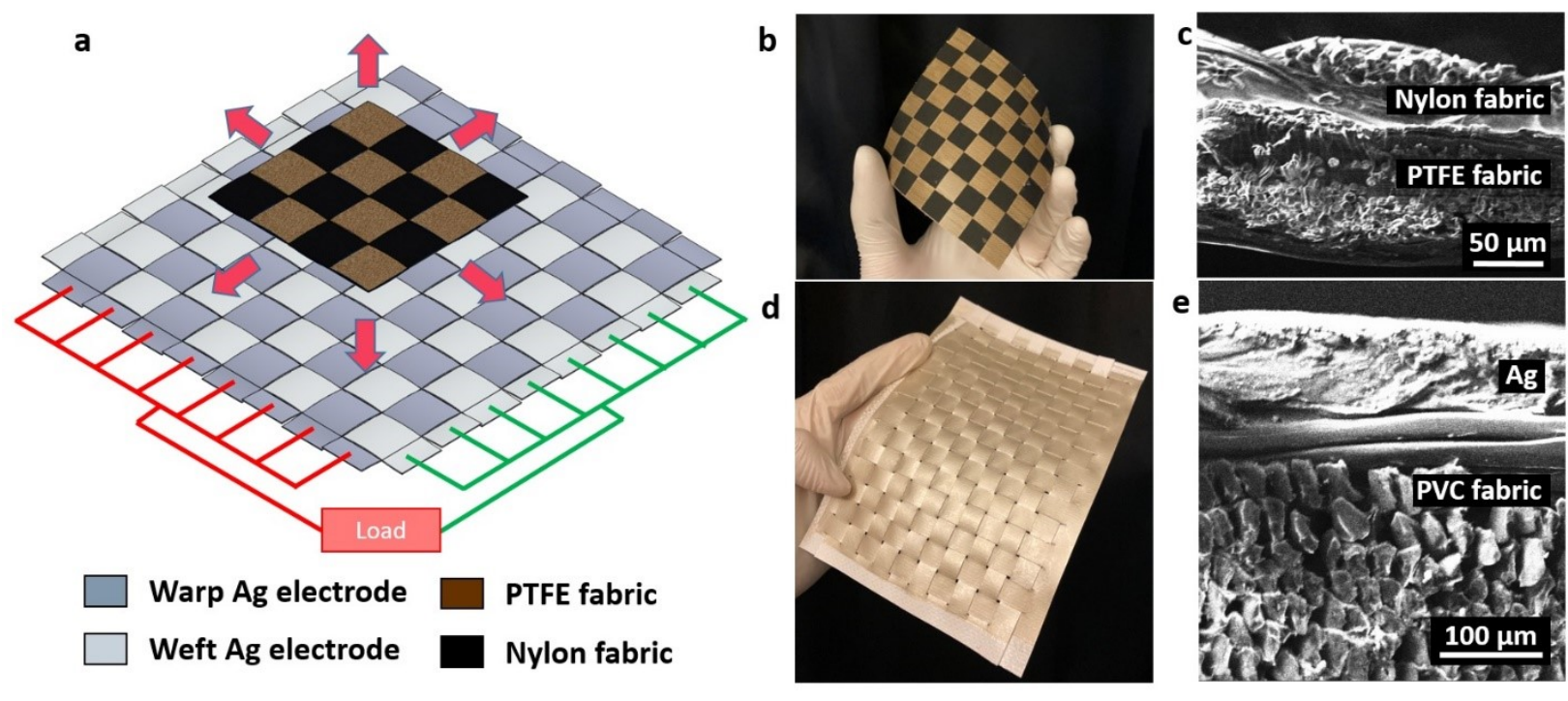

Fig. 1. Schematic illustration of (a) woven-TENG for $N=4$ with nylon fabric as positive material, PTFE fabric as negative material and woven Ag electrodes. (b) Photograph and (c) SEM image of top substrate of the woven-TENG comprising the PTFE and nylon fabric for $N=8$. (d) Photograph and (e) SEM image of bottom substrate of the woven-TENG comprising the Ag coated PVC fabric as the electrodes.

\subsection{Fabrication}

A schematic illustration of the woven-TENG is shown in Fig. 1a. The woven-TENG is composed of a top substrate comprising woven strips of positive and negative triboelectric materials and a bottom substrate serving as electrodes. The photograph and the SEM image of the top substrate are shown in Fig. $1 \mathrm{~b}$ and 1c, respectively. It was fabricated by cutting PTFE coated fibreglass fabric (PTFE fabric, Xinghoo) and adhesive nylon fabric (Hemline) into strips of the same size and weaving them together forming a checker-like pattern with a total size of $8 \mathrm{~cm} \times 8$ $\mathrm{cm}$. The photograph and the SEM image of the bottom substrate are shown in Fig. 1d and 1e, respectively. The PTFE fabric and the nylon fabric were chosen as the triboelectric materials, because PTFE and nylon are among the most negative and positive material according to the triboelectric series proposed by Zou et al. [28], respectively. Moreover, they are available in a commercial fabric form and have good properties for implementation in wearable devices, such as flexibility, lightweight, biocompatibility and washability. The electrodes were fabricated by firstly pre-baking two pieces of $450-\mu \mathrm{m}$-thick PVC coated polyester fabric (PVC fabric, VALMEX FR 
7546) at $120{ }^{\circ} \mathrm{C}$ for 15 minutes in a Carbolite box oven to eliminate outgassing from the PVC layer. Next, doctor blading was used to coat the PVC fabric with a 100- $\mu$ m-thick Ag ink (Fabinks TC-C4001) and curing at a temperature of $120{ }^{\circ} \mathrm{C}$ for 15 minutes. The Ag coated PVC fabrics were then cut into strips of the same size with a 1-mm gap between each strip and leaving one end uncut. Finally, the two Ag coated PVC fabrics are woven together. The uncut ends enable the warp strips and weft strips to be electrically connected forming separate warp and weft electrodes. The 1-mm gap ensures the warp and weft electrodes are electrically isolated after weaving. The top and the bottom substrates were produced with various numbers of elements $(N)$ on each side with $N$ equalling 2, 4, 6 and 8 with strip widths of $4 \mathrm{~cm}, 2 \mathrm{~cm}, 1.33 \mathrm{~cm}$ and $1 \mathrm{~cm}$, respectively, thereby maintaining a constant overall area.

\subsection{Working principle}

The operating mechanism of the woven-TENG for $N=2$ is revealed in Fig. 2. The top insets show the bird's eye view of each location of the top layer relative to the bottom woven electrodes, while the bottom drawings show the corresponding cross-sectional view at the position of the dashed blue line shown in the top inset. When the top substrate slides on the bottom Ag electrodes and make a contact with them. The PTFE fabric and the nylon fabric will be negatively and positively charged, respectively. The same amount of charge with the opposite polarity is transferred to the electrodes due to the contact electrification effect. Electrons, ions and material transfer have been proposed to be the charge species responsible for the electrification [29-31]. In the beginning, the PTFE fabric and the nylon fabric fully overlap with the warp and the weft electrode, respectively (Fig. 2(i)). In this state, there is no charge transfer between the electrodes. They are in electrostatic equilibrium since the number of positive and negative charges on their surfaces are equal. In Fig. 2(ii), the top substrate is sliding further to the right-hand side along the waft electrode. In this state, the electrons flow from the weft to the warp electrode through the load, because the electric potential of the weft electrode decreases, while that of the warp electrode increases due to the presence of the PTFE fabric (negative) and the nylon fabric (positive) on the weft and the warp electrode, respectively. When the PTFE fabric and the nylon fabric fully overlap with the weft and the warp electrode, respectively (Fig. 2(iii)), another electrostatic equilibrium is reached and the charge transfer stops. In the $4^{\text {th }}$ state (Fig. 2 (iv)), the upper substrate is moving back to the left-hand side. The electric potential of the warp electrode decreases, while that of the 
weft electrode increases, resulting in a current flow through the load from the weft to the warp electrode. The current keeps flowing until the potential gradient terminates when the next full overlap occurs. When the upper substrate continually slides in cycles, an alternating current is generated through the load. The same effect occurs when the upper substrate moves perpendicularly along the warp electrode. The output of the woven-TENG according to the movement of the upper substrate at different angles with respect to the weft electrode (x-axis) will be further discussed in section 3.3.

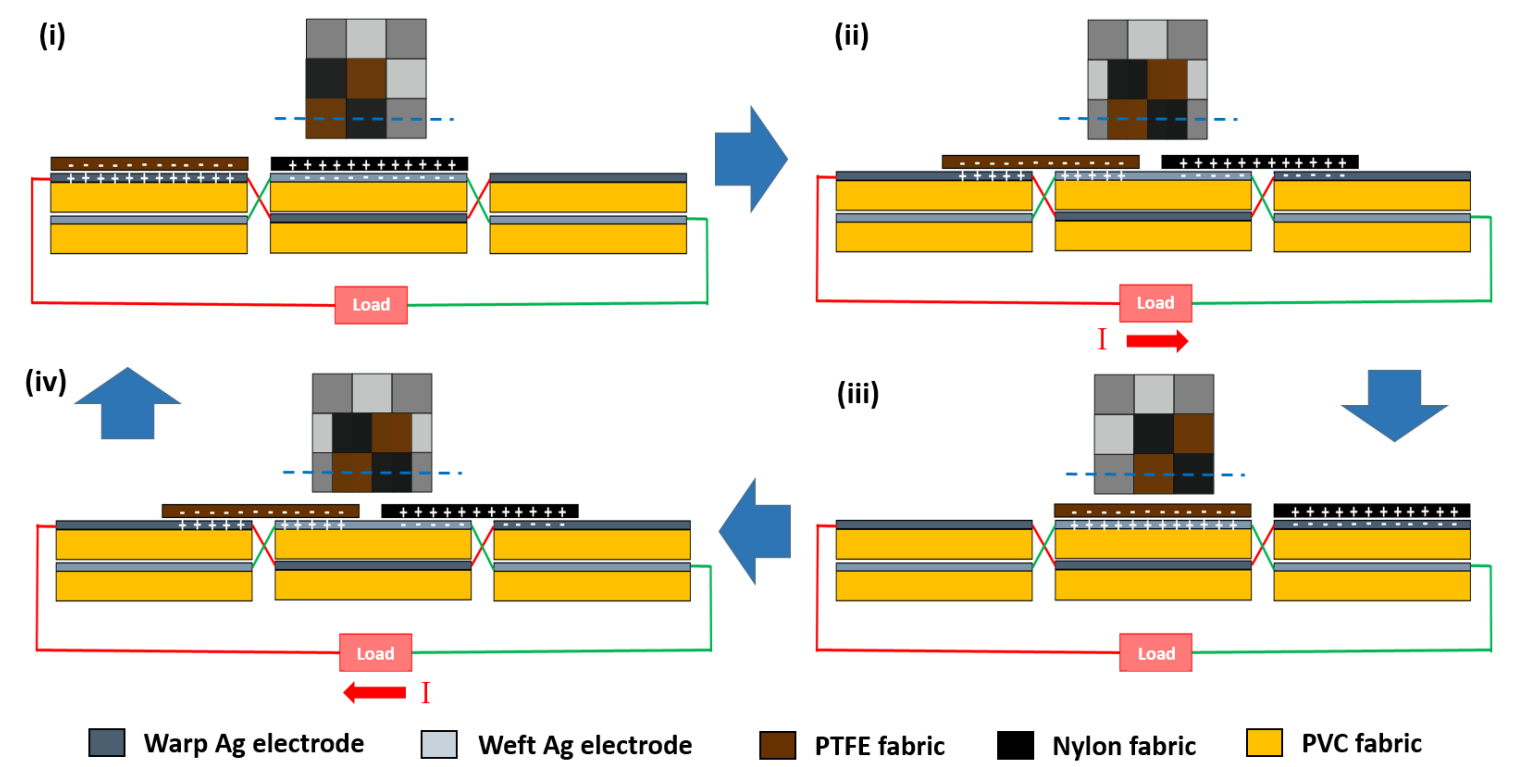

Fig. 2. Schematic illustration of the operating mechanism of woven-TENG for $N=2$.

\subsection{Characterisation and measurement}

The experiments were performed using a belt-driven linear actuator, which can produce periodically sliding motion between the top and the bottom substrates with a constant contact force thereby ensuring a repeatable experimental measurement process. For initial testing purposes, the top and the bottom substrates were first attached to a piece of $1 \mathrm{~mm}$-thick fabric, then an acrylic sheet. The fabric allows the triboelectric material to conform to the electrodes and thus maintain the effective contact area of the triboelectric materials, while the acrylic sheet enables consistent and repeatable testing but would not be required in an actual application (see section 3.6). If not specified otherwise, all measurements were carried out in an environmental chamber at an oscillation frequency of $2 \mathrm{~Hz}$, an amplitude of $40 \mathrm{~mm}$, a contact force of $5 \mathrm{~N}$, a temperature of 25 
${ }^{\circ} \mathrm{C}$ and a humidity of $25 \% \mathrm{RH}$. Open-circuit-voltage $\left(V_{O C}\right)$ and Short-circuit-current $\left(I_{S C}\right)$ were measured using an oscilloscope (Agilent DSO3062A) at $1 \mathrm{G} \Omega$ load and a DC power analyzer (Agilent N6705B), respectively. For the capacitor charging measurement, the output of the wovenTENGs was first rectified using a commercial full-wave bridge rectifier (Diodes Inc DF10M) and then used to charge a $10-\mu \mathrm{F}$ electrolytic capacitor. During charging, the capacitor voltages $\left(V_{C}\right)$ were measured using a digital multimeter (Tenma 72-7780) and oscilloscope (Agilent DSO3062A). Surface charge densities of the triboelectric materials were measured using a bespoke Faraday cup connected to an electrometer (Keithley 6514), as shown in Fig. S1 in the supplementary material. COMSOL Multiphysics software was employed for the $V_{O C}$ simulation.

\section{Result and discussion}

\subsection{Effect of the number of elements per each side of the top substrate (N)}

\subsubsection{Simulation and theoretical calculation}

For the theoretical investigation of the effect of element number per each side of the top substrate on the outputs of woven-TENG, the peak $V_{O C}$ of the woven-TENGs for $N=2,4,6$ and 8 were simulated using COMSOL. Electrostatics physics with a normal free triangular mesh was used to simulate the maximum $V_{O C}$ at a stationary state when the positive and negative triboelectric materials fully overlap with the electrodes. Fig. 3a demonstrates a magnified cross-section simulation result of the electric potential distribution for the woven-TENG with $N=2$. The stack of the materials on the left-hand side from top to bottom is PTFE, Ag warp electrode, PVC, Ag weft electrode and PVC, respectively. The stack of the materials on the right-hand side from top to bottom is nylon, Ag weft electrode, PVC, Ag warp electrode and PVC, respectively. The surface charge density of the PTFE and nylon in the simulation were pre-defined as $-19.24 \mu \mathrm{C} / \mathrm{m}^{2}$ and $11.16 \mu \mathrm{C} / \mathrm{m}^{2}$, respectively. These values were obtained from measurements of the PTFE fabric and the nylon fabric using the Faraday's cup (Fig. S1). The top-left and the bottom-right electrode were electrically connected with the same floating potential, similar to the bottom-left and the topright electrode. Due to the presence of the negative charges on the PTFE surface on the left-hand side, the electric potential on the warp electrode is $-19633 \mathrm{~V}$, while the underlying weft electrode has a higher potential of $-19537 \mathrm{~V}$. The reasons for this are firstly, the electric field from the negative charge is shielded by the top electrode [32]. Secondly, the bottom electrode is located 
further from the negatively charged surface and electric potential increases with increasing distance from negative point charges according to Eq. 1 below:

$$
V_{E}=\frac{1}{4 \pi \varepsilon_{0}} \frac{Q}{r}
$$

where $V_{E}$ is the electric potential, $Q$ is the point charge, $r$ is the distance from the point charge and $\varepsilon_{0}$ is the permittivity of vacuum. Thirdly, the positively charged surface on the right-hand side induces a positive electric potential at the top-right electrode, which is electrically connected to the bottom-left electrode. However, the net electric potential of both electrodes is negative because of the dominance of the negative surface charge density of the PTFE over the nylon. The $V_{O C}$ can be determined from the difference of the electric potentials between the electrodes and plotted in Fig. 3b. It decreases with increasing element number, which can be explained by Eq. 2 below [17]:

$$
V_{o c}=\frac{\Delta \sigma_{s c} \cdot S}{C}
$$

where $\Delta \sigma_{s c}$ is the short-circuit transferred charge density, $S$ is the total area of the electrodes and $C$ is the capacitance between the electrodes. $\Delta \sigma_{s c}$ and $S$ are consistent for all $N$ but $C$ rises when the electrodes are divided into small elements, therefore $V_{O C}$ reduces with increasing element number [17]. The simulated $V_{O C}$ of the woven TENG with $N=2,4,6$ and 8 are $95.6 \mathrm{~V}, 94.7 \mathrm{~V}$, 93.8 and 92.9 respectively.

The theoretical calculation of the corresponding average $I_{S C}$ for FT-mode TENG with positive and negative triboelectric material was performed based on electrostatic induction and the charge conservation principle. This can be expressed as Eq. 3 below [28]:

$$
I_{S C}=\frac{A_{M} \cdot \Delta \sigma_{q}}{\Delta t}
$$

where $A_{M}$ is the total area of the triboelectric materials, $\Delta \sigma_{q}$ is the difference of the surface charge density between the triboelectric materials and $\Delta t$ is the time taken of the upper substrate to move from one electrode element to the next electrode element. As before, a constant surface charge density of the PTFE fabric of $-19.24 \mu \mathrm{C} / \mathrm{m}^{2}$ and the nylon fabric of $11.16 \mu \mathrm{C} / \mathrm{m}^{2}$ are used in the calculation. With a constant $A_{M}$ of $64 \mathrm{~cm}^{2}$, a constant $\Delta \sigma_{s c}$ of $30.40 \mu \mathrm{C} / \mathrm{m}^{2}$ and a different $\Delta t$ for $N=2,4,6$ and 8 , the calculated average $I_{S C}$ are $0.39 \mu \mathrm{A}, 0.78 \mu \mathrm{A}, 1.17 \mu \mathrm{A}$ and $1.56 \mu \mathrm{A}$, respectively (Fig. 3b). Since $\Delta t$ reduces with increasing element number, the $I_{S C}$ grows. 


\subsubsection{Experiment}

The electrical experiments for the woven-TENG with $N=2,4,6$ and 8 were carried out using the belt-driven linear actuator under the conditions specified in section 2.3. The transient $V_{O C}$ and $I_{S C}$ are represented in Fig. $3 \mathrm{c}$ and $3 \mathrm{~d}$, respectively. Their corresponding peak and RMS values are plotted in Fig. 3e and 3f, respectively. Both the peak and RMS $V_{O C}$ slightly decline with rising element number. The peak $V_{O C}$ varies between $109 \mathrm{~V}$ for $N=2$ to $102 \mathrm{~V}$ for $N=8$. These are in good agreement with the simulation results. The RMS $V_{O C}$ also shows a small reduction from $62.9 \mathrm{~V}$ for $N=2$ to $61.5 \mathrm{~V}$ for $N=8$. In contrast, the peak and RMS $I_{S C}$ show a substantially increasing trend. The peak $I_{S C}$ rises from $1.29 \mu \mathrm{A}$ for $N=2$ to $2.99 \mu \mathrm{A}$ for $N=8$ and the RMS $I_{S C}$ ranges between $0.81 \mu \mathrm{A}$ and $1.77 \mu \mathrm{A}$, which matches well with the simulated average $I_{S C}$.

Fig. 3g demonstrates the dependence of RMS values of the voltage $\left(V_{R M S}\right)$, current $\left(I_{R M S}\right)$ and power $\left(P_{R M S}\right)$ on the external load resistance for the woven-TENG with $N=8$. The $V_{R M S}$ climbs with rising load resistance and reaches a maximum value of $62.9 \mathrm{~V}$ at a load resistance of $2 \mathrm{G} \Omega$, whereas the $I_{R M S}$ reduces with increasing load resistance. It starts from a maximum value of 1.77 $\mu \mathrm{A}$ at no load and falls to zero at the maximum load. The $P_{R M S}$ values are derived from the product of $V_{R M S}$ and $I_{R M S}$. The maximum $P_{R M S}$ of $34.8 \mu \mathrm{W}$ is obtained at a load resistance of $50 \mathrm{M} \Omega$, corresponding to a maximum RMS power density of $5.43 \mathrm{~mW} / \mathrm{m}^{2}$. The $P_{R M S}$ for the different $N$ are compared in Fig. 3h. The graph for $N=2,4,6$ and 8 reach a peak of $17.0 \mu \mathrm{W}, 30.8 \mu \mathrm{W}, 34.2$ $\mu \mathrm{W}$ and $34.8 \mu \mathrm{W}$ at the same load resistance of $50 \mathrm{M} \Omega$, respectively. Compared to the two previously reported TENGs which can also generate power from all sliding directions (Guo et al. [20] with the maximum power density of $1.9 \mathrm{~W} / \mathrm{m}^{2}$ and Xia et al. [21] with the maximum power density of $2.1 \mathrm{~W} / \mathrm{m}^{2}$ ), the power generated by the woven-TENG seems to be very low. However, these two TENGs are made of rigid materials and the claimed power is the instantaneous peak power output, which does not reflect the true output of the device. Comparing the peak output current at the same frequency of $2 \mathrm{~Hz}$, both TENGs produces a peak $I_{S C}$ of around $3-4 \mu \mathrm{A}$, which is comparable with the peak $I_{S C}$ of $2.99 \mu$ A produced by the woven-TENG $(N=8)$. 

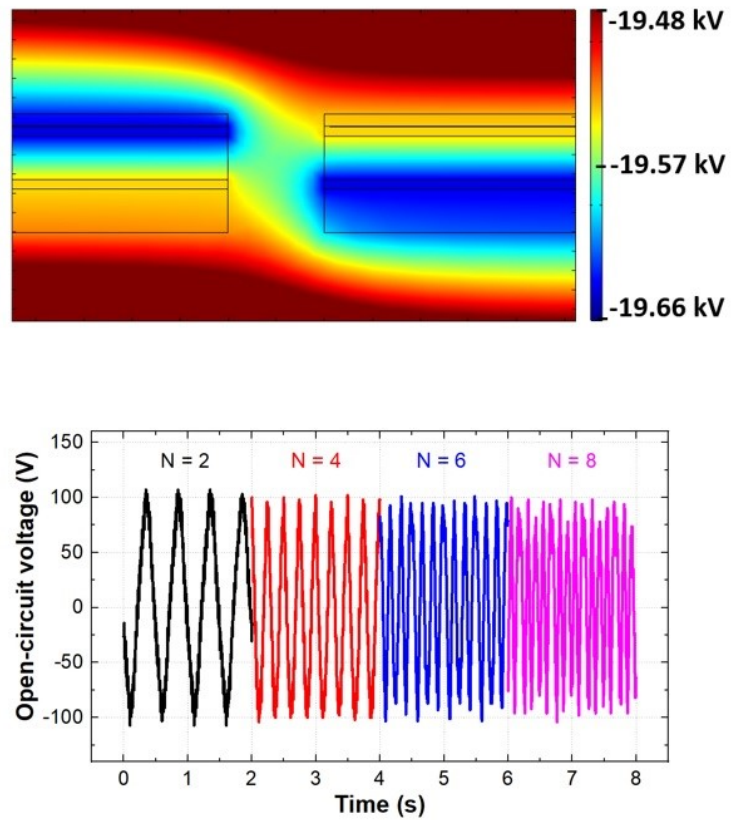

e

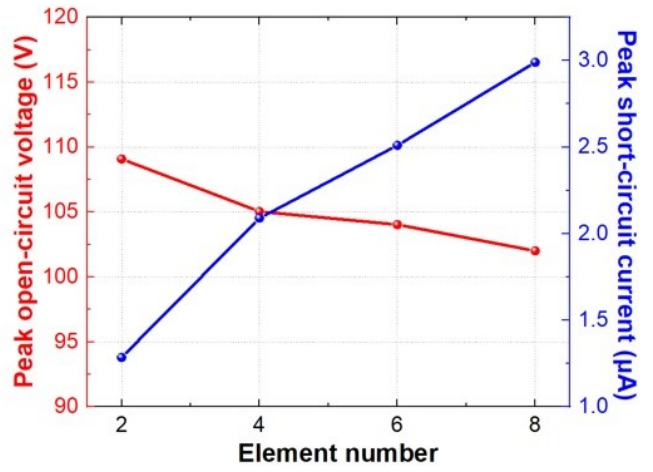

g

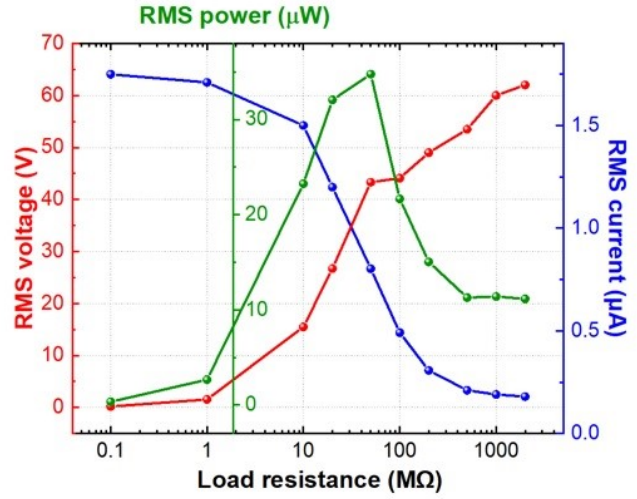

b

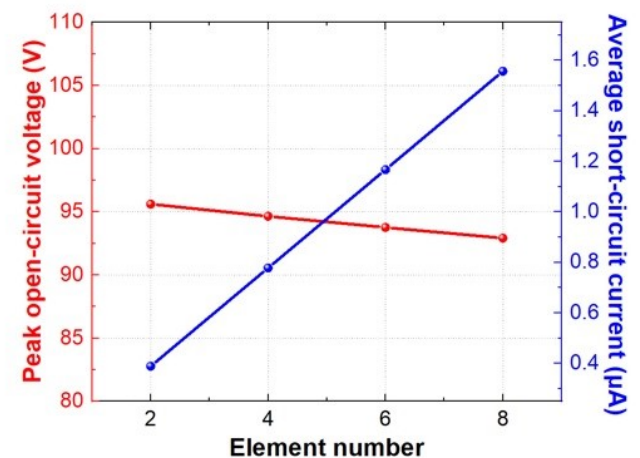

d


Fig. 3. (a) Simulated electric potential distribution of woven-TENG for $N=2$. (b) Simulated peak $V_{O C}$ and calculated average $I_{S C}$ for different $N$. Experimental results of transient (b) $V_{O C}$ and (c) $I_{S C}$. (e) Corresponding experimental peak $V_{O C}$ and $I_{S C}$ for different $N$. (f) Corresponding experimental RMS $V_{O C}$ and $I_{S C}$ for different $N$. (g) Dependence of the RMS voltage, current and power on the external load resistance for the woven-TENG with $N=8$. (h) Load-dependent RMS power for the woven-TENG with different $N$. 


\subsection{Effect of the positive and negative triboelectric material}

To demonstrate that the implementation of the positive and negative triboelectric material substantially enhances the outputs of woven-TENGs, the woven-TENG with PTFE/nylon $(N=4)$ was compared with checker-like patterned TENGs with only one negative material (PTFE) and with only one positive material (nylon), tested under the same conditions. The photographs of the TENGs with single triboelectric material are illustrated in Fig. S2 in the supplementary material. As shown in Fig. 4a, the transient $V_{O C}$ of the woven-TENG with PTFE/nylon are 1.3 times and 2.6 times greater than that of the TENG with PTFE and the TENG with nylon, respectively, while the transient $I_{S C}$ of the woven-TENG improves by a factor of 1.3 and 3.0 respectively (Fig. 4b). The $P_{R M S}$ of the woven-TENG peaks at a maximum value of $30.8 \mu \mathrm{W}$, which is 2.2 and 12.4 times higher than that of the TENG with PTFE and the TENG with nylon, respectively (Fig. 4c). The $V_{C}$ of a 10- $\mu \mathrm{F}$ capacitor charged by the output of the woven-TENG, the TENG with PTFE and the TENG with nylon are plotted in Fig. $4 \mathrm{~d}$. After $600 \mathrm{~s}$ of charging, the $V_{C}$ reaches $31.9 \mathrm{~V}, 22.6 \mathrm{~V}$ and $9.16 \mathrm{~V}$, respectively.
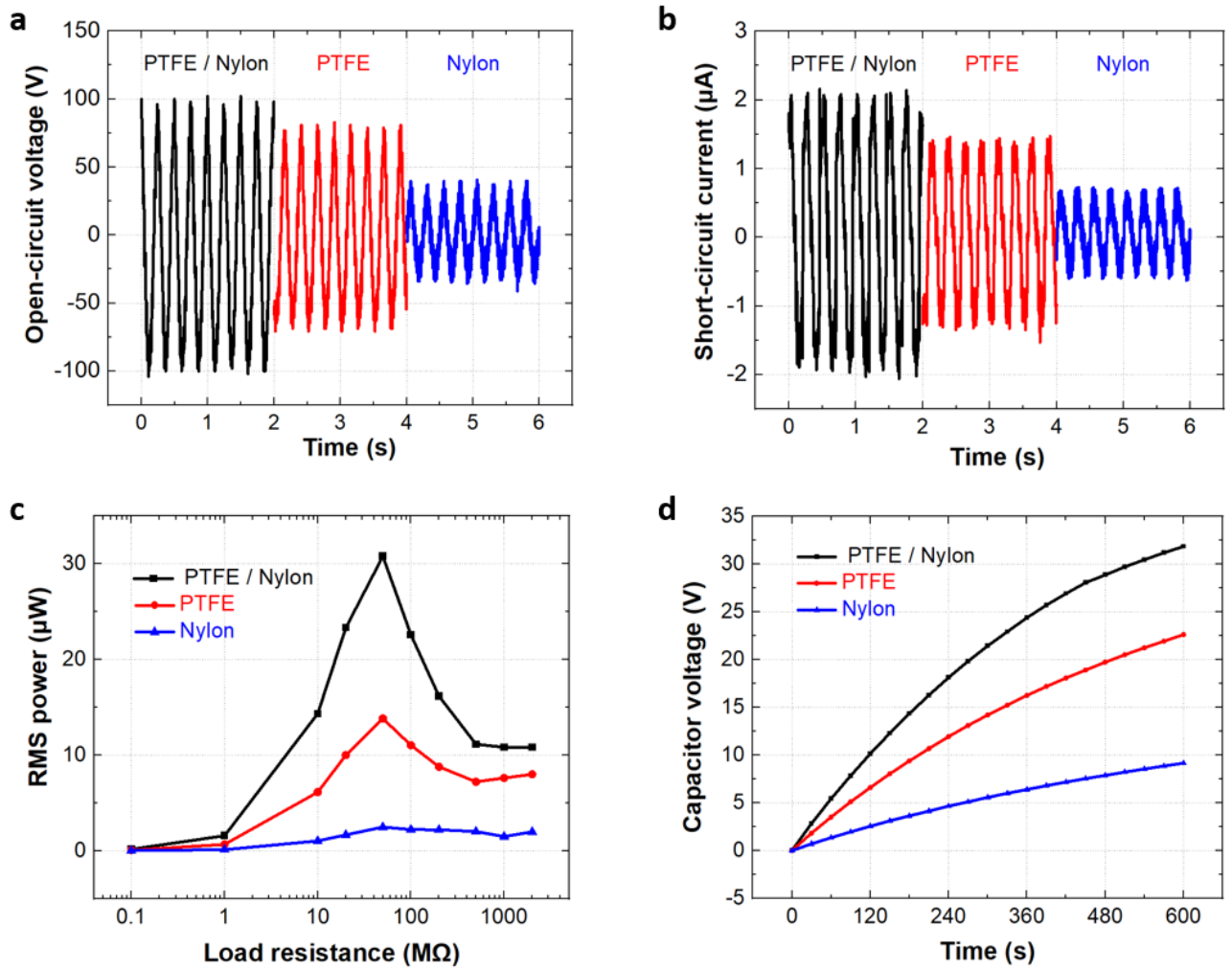
Fig. 4. Transient (a) $V_{O C}$ and (b) $I_{S C}$ for the woven-TENG with PTFE/nylon $(N=4)$, a checker-like patterned TENG with one negative material (PTFE) and a checker-like patterned TENG with one positive material (nylon). (c) Load-dependent RMS power and $(d)$ transient capacitor voltage $\left(V_{C}\right)$ for different types of TENGs.

\subsection{Direction dependence}

One major advantage of the proposed woven-TENG over the grating structured TENG is the ability to harvest energy from all planar sliding directions. Firstly, a model shown in Fig. 5a was applied for the theoretical calculation of the electrical outputs of a simple woven-TENG for $N=$ 2. The top transparent substrate comprises a nylon fabric marked in blue and a PTFE fabric marked in red. The bottom substrate consists of a warp electrode marked in white aligned in y-direction and a weft electrode aligned in x-direction (Fig. 5a (i)). Fig. 5a (ii) shows the initial state when the top substrate fully overlaps with the electrodes. In Fig 5a (iii), the top substrate moves at an angle $\theta$ relative to the $\mathrm{x}$-axis. The top substrate is transparent in this illustration and when the nylon fabric overlaps with the weft electrode, the overlapping areas become green. Likewise, when the PTFE fabric overlaps with the weft electrode, the areas become orange. In the initial state (ii), electrons accumulate at the warp electrode due to the presence of positive charges on the nylon surface. At the same time, electrons are repelled from the weft electrode due to the presence of negative charges on the surface of the PTFE fabric. In state (iii), the nylon fabric partly overlaps with the weft electrode (green areas) and the PTFE fabric partly overlaps with the warp electrode (red areas) resulting in an electron transfer from the warp to the weft electrode. The amount of the transferred electrons is proportional to the sum of the green and the red areas. Due to the symmetry of the elements, the sum of the green and the red areas are always equal. For this reason, the model can be simplified by considering only the green areas. The maximum sum of the green areas and thus the maximum transferred charges are obtained, when $\theta$ equals $0^{\circ}$ and $90^{\circ}$, while the minimum transferred charge of $50 \%$ of the maximum is obtained for $\theta$ equals $45^{\circ}$. These are illustrated in Fig. S3 in the supplementary material.

To determine a percentage of the transferred electrons for a given angle $\theta$ compared to the maximum output, the sum of the green areas $A$ in the zoom-in inset in Fig. 5a (iii) is calculated using the following equations:

$$
A=A_{1}+A_{2}=y(w-x)+x(w-y)
$$


where $A_{1}$ is the first green area, $A_{2}$ is the second green area, $w$ is the width of the element and $s$ is the travel distance of the top substrate from the initial state. Assuming that the nylon fabric element does not travel further than the adjacent electrode element, $x$ and $y$ can be expressed by Eq. 5 and 6 below:

$$
\begin{aligned}
& x=s \cos \theta, s \cos \theta \leq w \text { and } 0^{\circ} \leq \theta \leq 90^{\circ} \\
& y=s \sin \theta, s \sin \theta \leq w \text { and } 0^{\circ} \leq \theta \leq 90^{\circ}
\end{aligned}
$$

By substitution of Eq. 5 and 6 into Eq. 4, Eq. 4 can be rewritten as a function of $s$ below:

$$
A(s)=w s(\sin \theta+\cos \theta)-s^{2} \sin 2 \theta
$$

This function is a parabola function opening downward, which has a maximum at a travel distance $s_{\max }$. To find this, the first derivative of Eq. 7 is set equal to zero as below:

$$
A^{\prime}\left(s_{\max }\right)=w(\sin \theta+\cos \theta)-2 s_{\max } \sin 2 \theta=0
$$

After solving for $s_{\max }$, we obtain

$$
S_{\max }=\frac{w(\sin \theta+\cos \theta)}{2 \sin 2 \theta}
$$

By substitution of Eq. 9 into Eq. 7, the maximum area $A_{\max }$ can be expressed as Eq. 10 below:

$$
A_{\max }=\frac{w^{2}(\sin \theta+\cos \theta)^{2}}{4 \sin 2 \theta}
$$

The percentage of the transferred electrons $p$ in at an angle $\theta$ compared to the maximum transferred electrons can be calculated by the Eq. 11 below:

$$
p=\frac{A_{\max }}{w^{2}} \times 100=\frac{25(\sin \theta+\cos \theta)^{2}}{\sin 2 \theta}
$$

Considering the conditions in Eq. 5 and 6, $\theta$ must be in the range between $18.44^{\circ}$ and $71.56^{\circ}$. For $0^{\circ} \leq \theta<18.44^{\circ}$, the maximum area is obtained when $x=w$, thus $s_{\max }=\frac{w}{\cos \theta}$. For $71.56^{\circ}<\theta \leq 90^{\circ}$, the maximum area is obtained when $y=w$, thus $s_{\max }=\frac{w}{\sin \theta}$. With the same calculation method, we obtain 


$$
\begin{array}{lrl}
p & =(1-\tan \theta) \times 100, & 0^{\circ} \leq \theta<18.44^{\circ} \\
p=\frac{25(\sin \theta+\cos \theta)^{2}}{\sin 2 \theta}, & 18.44^{\circ} \leq \theta \leq 71.56^{\circ} \\
p=(1-\cot \theta) \times 100, & 71.56^{\circ}<\theta \leq 90^{\circ}
\end{array}
$$

Equation 12 are plotted in green for $0^{\circ} \leq \theta \leq 90^{\circ}$ in Fig. $5 \mathrm{~b}$ and compared to the experimental RMS $V_{O C}$ and $I_{S C}$ of the woven-TENG $(N=4)$ for $\theta=0^{\circ}, 15^{\circ}, 30^{\circ}, 45^{\circ}, 60^{\circ}, 75^{\circ}$ and $90^{\circ}$ on the same graph. The maximum outputs are produced for $\theta=0^{\circ}$, when the top substrate moves parallel to the PTFE fabric, followed by $\theta=90^{\circ}$, when the top substrate moves parallel to the nylon fabric and the minimum outputs of around the half of the maximum outputs are generated for $\theta=45^{\circ}$. These are in very good accordance with the theoretical calculation. Fig. 5c shows the $V_{C}$ of a 10$\mu \mathrm{F}$ capacitor charged by the output of the woven-TENG moving in different directions. The maximum $V_{C}$ is also obtained, when the $\theta=0^{\circ}$ and $\theta=90^{\circ}$, while the minimum $V_{C}$ is obtained, when the $\theta=45^{\circ}$.
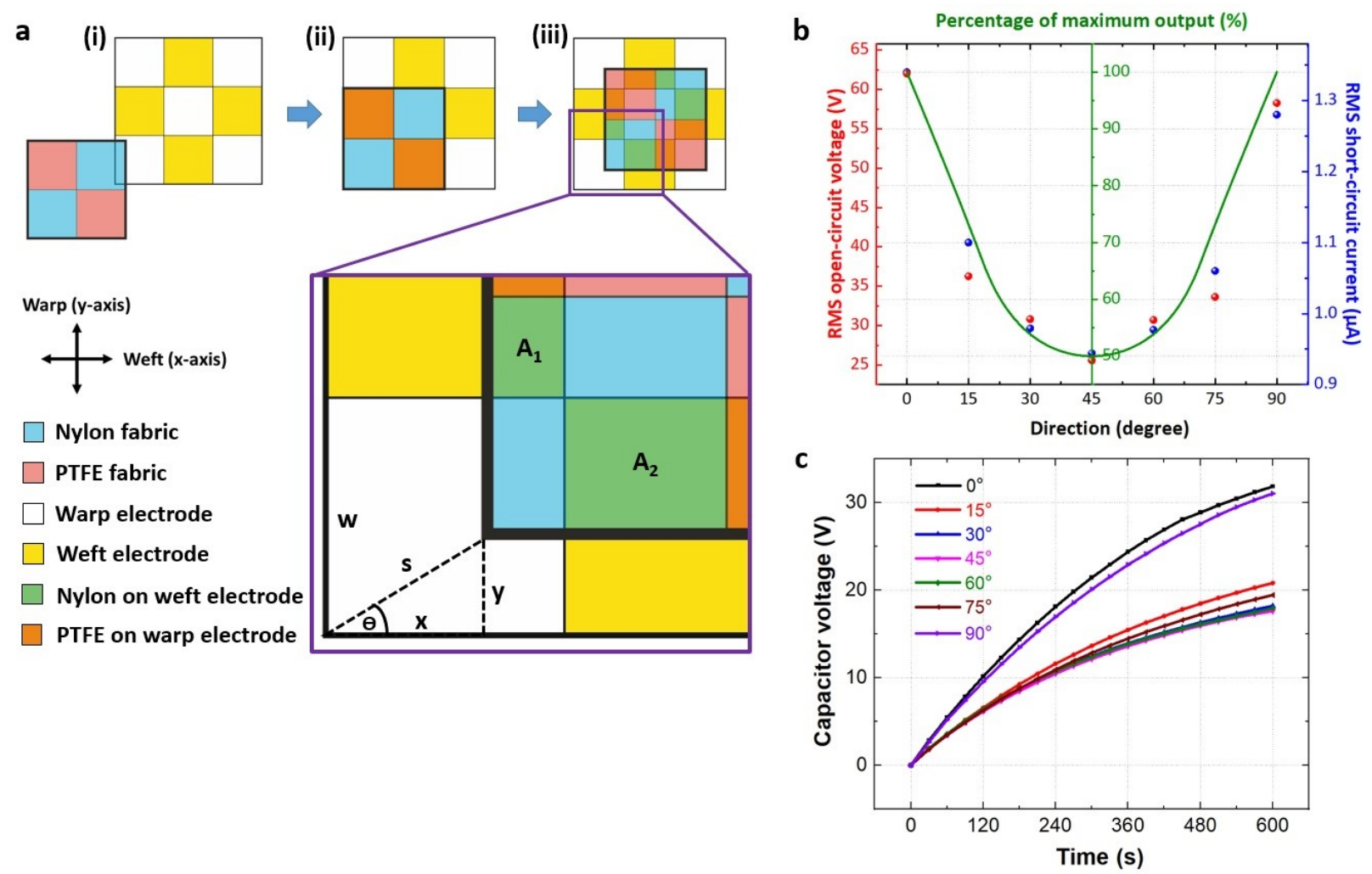

Fig. 5. (a) Schematic illustration applied for theoretical calculation of woven-TENG for $N=2$. (b) Calculated percentage of maximum output compared with experimental RMS $V_{O C}$ and RMS $I_{S C}$ as a function of sliding direction with respect to the x-axis. (c) Transient capacitor voltage for different sliding directions. 


\subsection{Oscillation frequency and contact force dependence}

The frequency dependence measurements were carried out for the woven-TENG with $N=4$ by varying the oscillation frequency of the linear actuator from $1 \mathrm{~Hz}$ to $3 \mathrm{~Hz}$ in steps of $0.5 \mathrm{~Hz}$ and keeping the other parameters constant (see section 2.3). The RMS $V_{O C}$ and $I_{S C}$ of the woven-TENG are plotted as a function of frequency in Fig. 6a. At first, the RMS $V_{O C}$ gradually grows with rising frequency and begins to saturate at a frequency of $2.0 \mathrm{~Hz}$. According to Eq.2, the $V_{O C}$ is explicitly independent on the frequency. However, the reason for this growth stems from a change in the surface charge density that increases with the frequency and saturates at a certain value. The RMS $I_{S C}$ increases linearly with increasing frequency since it is inversely proportional to the time $\Delta t$ according to Eq. 3, which declines with increasing frequency. The rectified output of the wovenTENG was used to charge a $10-\mu \mathrm{F}$ capacitor for $300 \mathrm{~s}$. The maximum $V_{C}$, capacitor charging current $\left(I_{C}\right)$ and capacitor charging power $\left(P_{C}\right)$ are calculated from the transient $V_{C}$, illustrated in Fig. S4a in the supplementary material. They all show an increased tendency. At a frequency of 3 $\mathrm{Hz}$, the $V_{C}, I_{C}$ and $P_{C}$ peak at $35.9 \mathrm{~V}, 1.3 \mu \mathrm{A}$ and $13.0 \mu \mathrm{W}$, respectively.

The force dependence measurements were performed for the woven-TENG with $N=4$ by changing the weight on top of the upper substrate from $1 \mathrm{~N}$ to $12 \mathrm{~N}$ in increments of $2 \mathrm{~N}$ and keeping the other parameters constant. The dependence of the RMS $V_{O C}$ and $I_{S C}$ on the contact force are shown in Fig. 6c. In the beginning, both of the outputs increase sharply with increasing contact force until they become essentially constant at a contact force over $7 \mathrm{~N}$. The transient $V_{C}$ as a function of contact force is shown in Fig. S4b. The corresponding maximum $V_{C}, I_{C}$ and $P_{C}$ for the different contact forces are represented in Fig. 6d. They show the same tendency as the RMS $V_{O C}$ and $I_{S C}$. The $V_{C}, I_{C}$ and $P_{C}$ saturate at around $30.9 \mathrm{~V}, 1.1 \mu \mathrm{A}$ and $10.1 \mu \mathrm{W}$, respectively. 

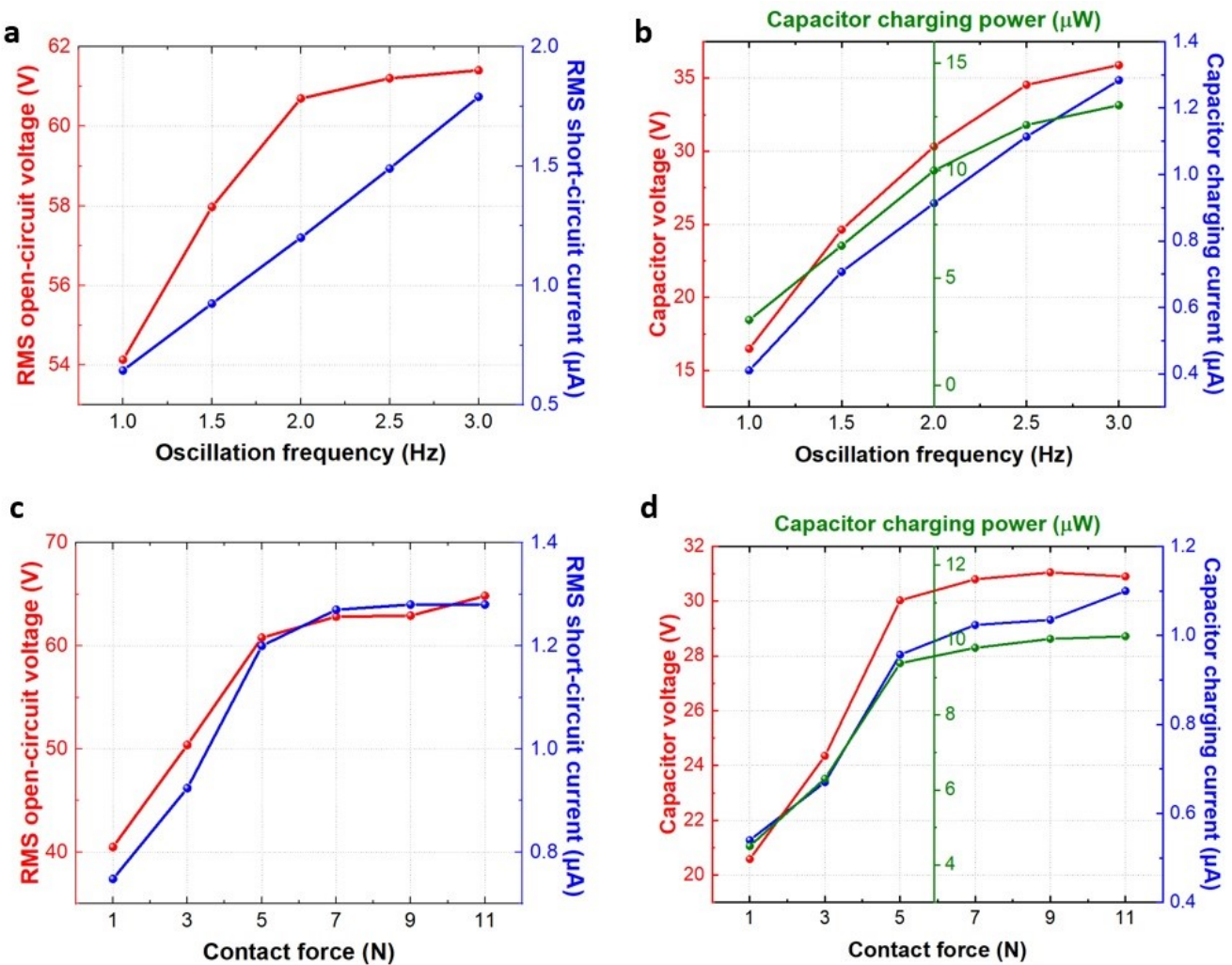

Fig. 6. (a) RMS $V_{O C}$ and $I_{S C}$ as a function of oscillation frequency. (b) Maximum $V_{C}, I_{C}$ and $P_{C}$ as a function of oscillation frequency. (c) RMS $V_{O C}$ and $I_{S C}$ as a function of contact force. (d) Maximum $V_{C}, I_{C}$ and $P_{C}$ as a function of contact force.

\subsection{Durability and washability}

Durability and washability are important properties that need to be tested for the practical utilisation of the woven-TENG in wearable devices. The top and the bottom substrate of the woven-TENG with $N=4$ were first rubbed against each other using the linear actuator under the condition specified in section 2.3. The recorded transient $V_{O C}$ and $I_{S C}$ are illustrated in Fig $7 \mathrm{a}$ and $7 \mathrm{~b}$, respectively. The $V_{O C}$ falls slightly from $102 \mathrm{~V}$ to $85 \mathrm{~V}$ after 40,000 operating cycles, while the $I_{S C}$ drops by $12.5 \%$. After hand-washing, the TENG output is restored to its original value. After 40,000 operating cycles, a minimal material transfer, abrasion of the Ag electrodes and fraying of the nylon fabric were observed. The fraying can be prevented by an adhesive layer on the backside of the nylon fabric. The photograph of the top and the bottom substrate after 40,000 operating cycles and during washing are shown in Fig. S5. The SEM image of the Ag electrode, PTFE fabric and nylon fabric before and after 40000 operating cycles, hand wash and machine washes are shown in Fig. S6 in the supplementary material. 
Fig. $7 \mathrm{c}$ and $7 \mathrm{~d}$ reveal the transient $V_{O C}$ and $I_{S C}$ after different numbers of washing cycles. The woven-TENG was initially hand-washed using water, detergent and brush, then dried at room temperature. After drying, the outputs of the woven-TENG was recorded. Next, it was placed in a laundry bag and washed using a commercial washing machine (Beko WME7247) for 49 min at a water temperature of $30^{\circ} \mathrm{C}$ and a spin speed of $1000 \mathrm{rpm}$ in hand-wash mode together with normal clothes and detergent. The woven-TENG was washed up to 4 times and the outputs were recorded after each wash. Both outputs remain stable indicating that the woven-TENG can survive this wash program. Additionally, the dependence of the output of the woven-TENG on the external humidity and temperature for wearable devices range is revealed in Fig. S7.
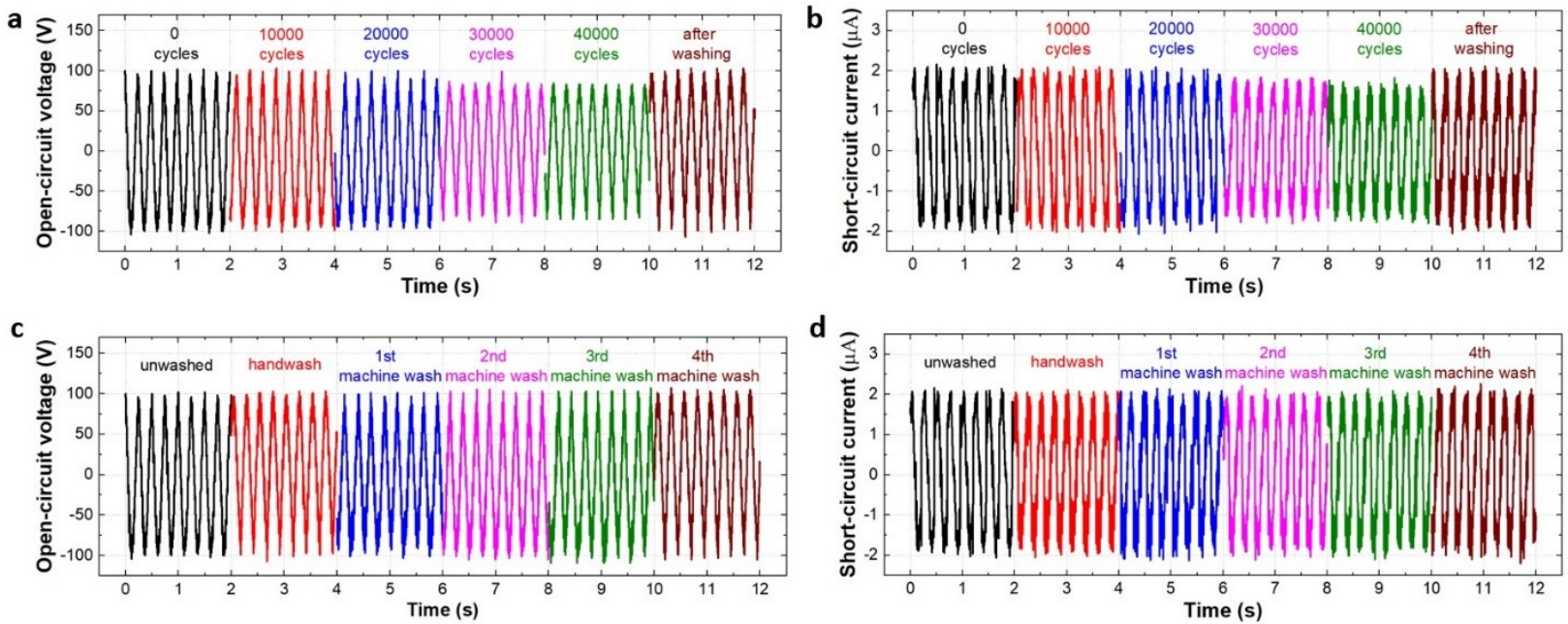

Fig. 7. Transient (a) $V_{O C}$ and (b) $I_{S C}$ for various operating cycles and after hand-washing. Transient (c) $V_{O C}$ and (d) $I_{S C}$ after different washing times.

\subsection{Applications in wearable electronics}

To illustrate a potential utilisation of the woven-TENG in wearable electronics, the wovenTENG with $N=8$ was embedded into a lab coat. The top substrate was attached to a lower sleeve and the bottom electrodes were attached to the hip and waist parts of the coat to harvest energy from walking and running, respectively (Fig. 8a). The energy is generated from the relative movement between the arm and torso, which does normally not occur in an exact straight line. For examples, the position of the arm movement can deviate from the direction of the electrode orientation by dozens of degrees during running depending on the physical characteristics of each person and the running speed and style. In all such cases, the device can still operate effectively 
and this is the major advantage of the woven-TENG presented here compared with TENGs with linear-grating structure, which require precise movement between the freestanding layer (arm) and the electrodes (torso). Fig. 8b represents the $V_{O C}$ produced during an arm swing frequency of around $0.75 \mathrm{~Hz}$ for walking and $2.30 \mathrm{~Hz}$ for running. A $V_{O C}$ of around $120 \mathrm{~V}$ is observed for both activities but the peaks for running are much more frequent. To confirm that this output stems from the device and is not parasitic signals from ambient, the parasitic signals have been therefore measured by directly rubbing the lab coat against the electrodes without the top triboelectric materials. The result shown in Fig. S8 demonstrates that the parasitic signals are very small and are insignificant compared to the signal produced from the woven-TENG and thus can be neglected. As shown in Fig. 8c and 8d, the output is rectified and used to charge different capacitors during walking and running, respectively. A 4.7- $\mu \mathrm{F}$ capacitor can be charged to a $V_{C}$ of $2.2 \mathrm{~V}$ and $5.2 \mathrm{~V}$ after $30 \mathrm{~s}$. This level of energy is sufficient to operate some wearable applications. To demonstrate this, the output of the woven-TENG was used to drive a wearable night-time warning indicator for pedestrians, a digital watch and a Bluetooth transceiver. The circuit diagrams for all applications are exhibited in Fig. S9 in the supplementary material. Additionally, since the device is attached to the coat, which is flexible and conformal, the influence of deformation on electrode conductivity is investigated and shown in Fig. S10.

Regarding the night-time warning indicator, the AC output of the woven-TENG can be directly used to flash 42 forward bias LEDs and 42 reverse bias LEDs aligning in a sign "STOP" for warning upcoming vehicles of the pedestrians at night (Fig. 8e and Video 1). Regarding the watch, the output is rectified and used to charge a $10-\mu \mathrm{F}$ capacitor. When the $V_{C}$ reaches $1.3 \mathrm{~V}$, the watch is turned on and can then be continuously powered (Fig. 8f and Video 2). Regarding the Bluetooth transceiver, the output is used to power an internet of things sensor node based on a microcontroller unit (Arm Cortex-M3) and a Bluetooth low energy (BLE) transceiver integrated in a system on chip (SoC). The rectified output is used to charge a $100-\mu \mathrm{F}$ capacitor up to $4.0 \mathrm{~V}$, which takes approximately 5 min when running. Next, the transceiver is turned on. An identification packet is then transmitted to a BLE gateway. The $V_{C}$ and the transceiver current $I_{T}$ consumed by the node are shown in Fig. 8g. The lower magnified inset reveals that the harvested energy could sustain the node's operation for almost $2 \mathrm{~s}$ with over 17 successful transmissions taking place in that period, observed through the 5-12 mA duty-cycled current spikes. The transmitted packet, at 1 $\mathrm{mW}$ RF power level, can be received by a standard BLE gateway at a distance of over $100 \mathrm{~m}$ away 
from the user, such as a smartphone shown in the top-left inset. In addition, wearable BLE nodes can be used as battery-free indoor localization beacons based on the relative signal strength [33].

Moreover, the woven-TENG can also be implemented as a sensing device. To demonstrate this, a pedometer that can be interfaced with a simple low-power microcontroller was built. A passive peak detect and hold circuit was implemented using a shunt RC filter. The values of the resistor and the capacitor were chosen as $8.2 \mathrm{M} \Omega$ and $264 \mathrm{nF}$, to control the delay of the signal for correct step detection. The voltage across the capacitor is measured using a microcontroller's analogue to digital converter every $300 \mathrm{~ms}$ and compared against a predetermined threshold to detect the walking or running steps (Fig. 8h and Video 3). 
a

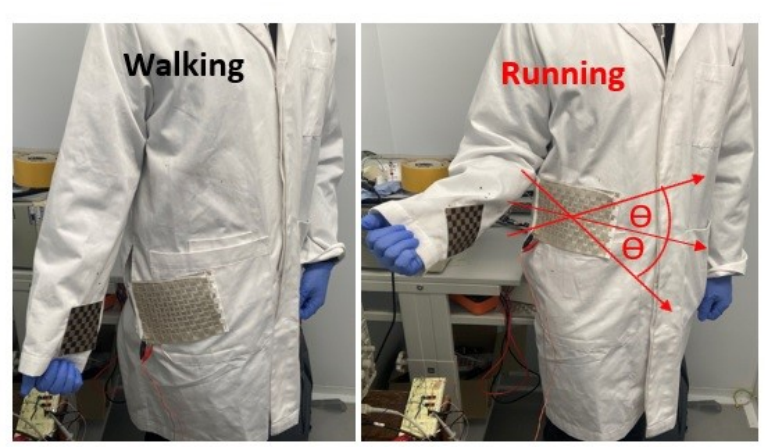

C

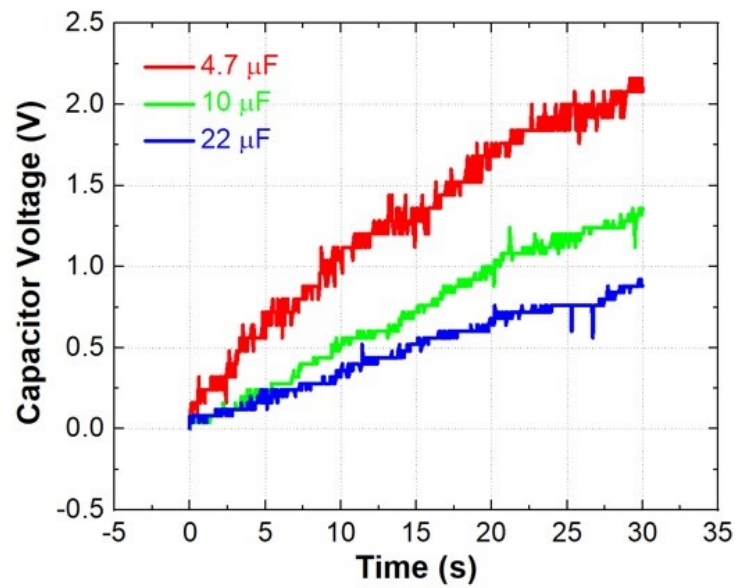

e



f

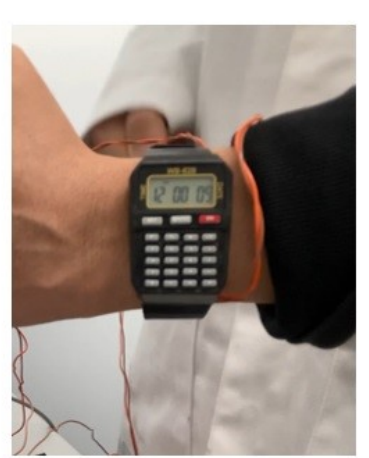

h

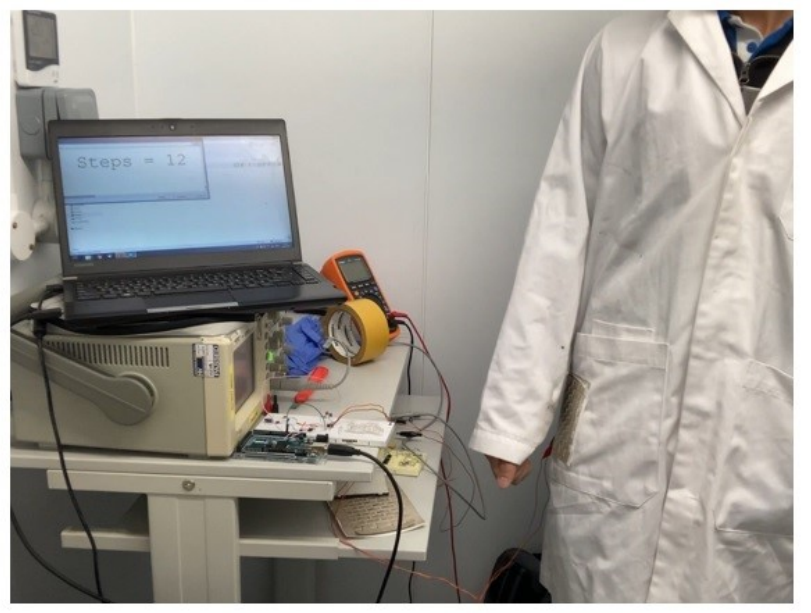

b

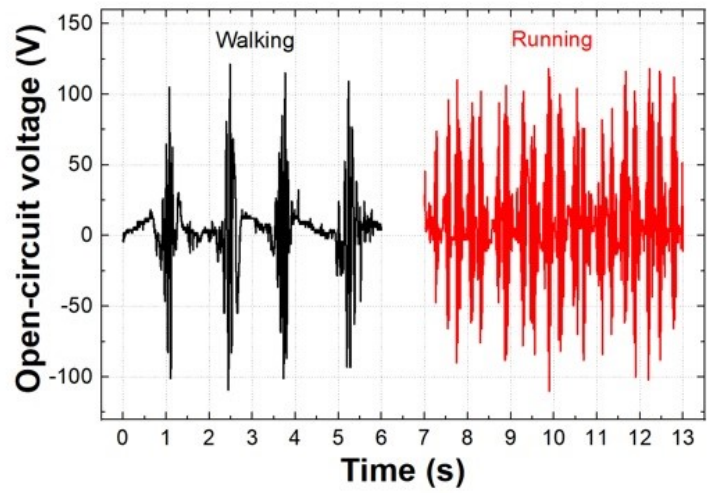

d

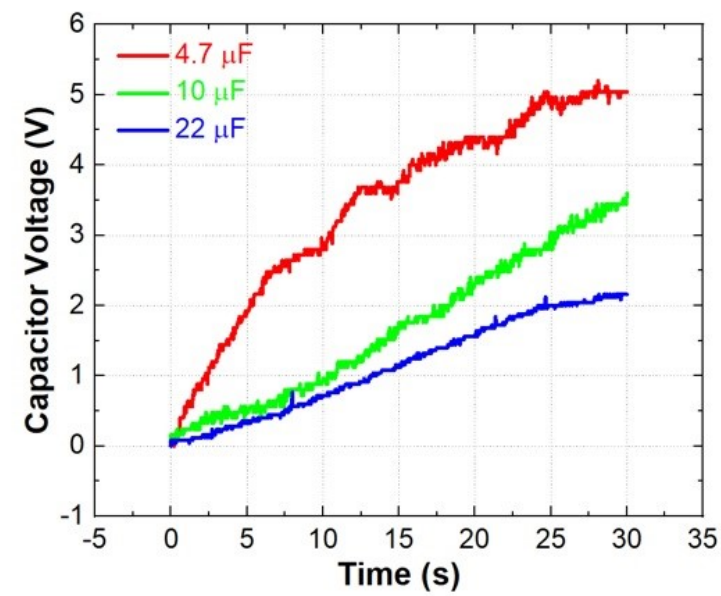

g

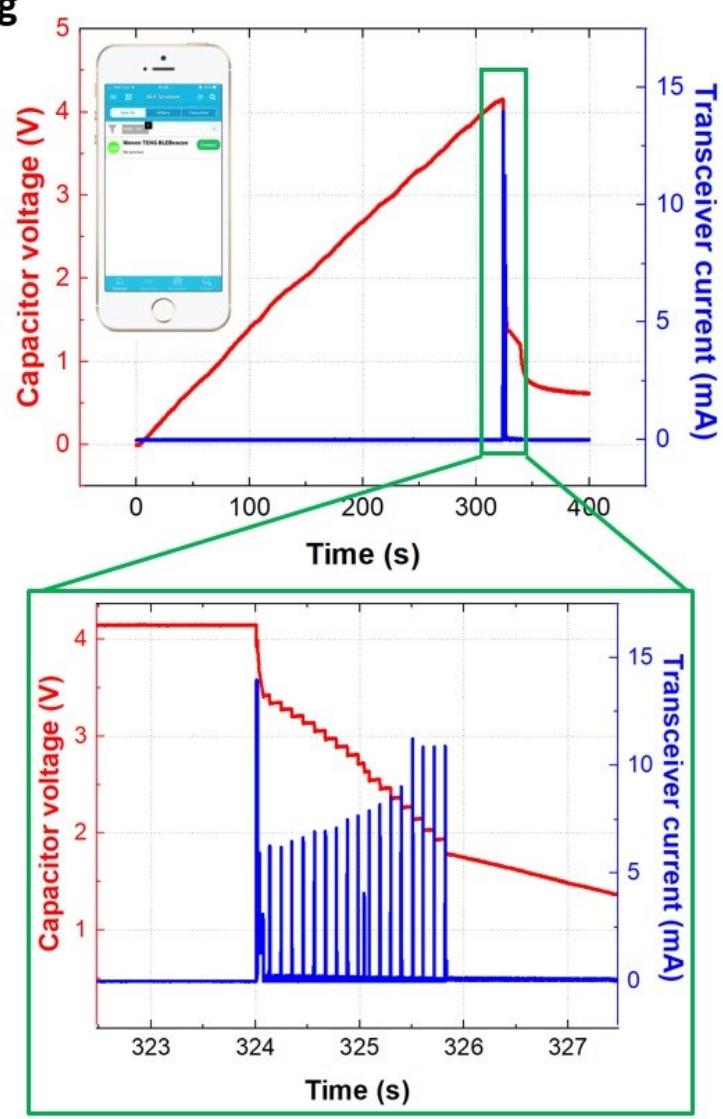


Fig. 8. (a) Photographs of woven-TENG embedded into a lab coat for harvesting energy from running and walking. (b) Transient $V_{O C}$ during running and walking. Transient $V_{C}$ for different capacitors charged during (c) running and (d) walking. Photograph of (e) wearable night-time warning indicator for pedestrians and (f) digital watch powered by the output of woven-TENG during running. (g) Transient $V_{C}$ and $I_{T}$ of a Bluetooth transceiver and microcontroller SoC driven by the woven-TENG during running. The top-left inset shows a Bluetooth advertisement received by a smartphone. The lower inset shows a zoom-in transient $V_{C}$ and $I_{T}$ of the area marked in green. (h) Photograph presenting the use of the woven-TENG as a sensor for step counting via arm motion (pedometer).

\section{Conclusion}

A novel textile-based triboelectric nanogenerator with woven strips of positive and negative triboelectric material (woven-TENG) designed to operate at all angles of relative sliding displacement has been demonstrated and characterised. The woven electrodes are made of Ag coated PVC fabric. The nylon fabric and PTFE fabric have been shown to be practical positive and negative triboelectric materials respectively for e-textile applications. The checker-like pattern can harvest energy from arbitrary sliding directions when operated as a freestanding triboelectric-layer TENG. The maximum output is produced, when the triboelectric materials slide parallel to the electrodes, whereas the minimum energy output (equal to half the maximum output) is generated when the triboelectric materials move diagonally at an angle of $45^{\circ}$ with respect to the alignment of the electrodes. The use of both positive and negative triboelectric material enhances the power of the woven-TENG by 2.2 times compared to the TENG with single triboelectric material.

\section{Acknowledgements}

The authors gratefully acknowledge the Engineering and Physical Sciences Research Council (EPSRC) for supporting this research with grant reference EP/P010164/1. The work of Steve Beeby was supported by the Royal Academy of Engineering under the Chairs in Emerging Technologies Scheme.

\section{Appendix A. Supplementary material}

The supplementary data associated with this article can be found in the online version at http://dx.doi.org/...

\section{References}

[1] F.-R. Fan, Z.-Q. Tian, Z. Lin Wang, Flexible triboelectric generator, Nano Energy. 1 (2012) 328-334. doi:10.1016/j.nanoen.2012.01.004.

[2] S. Wang, L. Lin, Z.L. Wang, Nanoscale-triboelectric-effect enabled energy conversion for sustainable powering of portable electronics, Nano Lett. 12 (2012) 6339-46. 
doi:10.1021/nl303573d.

[3] G. Zhu, Z.H. Lin, Q. Jing, P. Bai, C. Pan, Y. Yang, Y. Zhou, Z.L. Wang, Toward largescale energy harvesting by a nanoparticle-enhanced triboelectric nanogenerator, Nano Lett. 13 (2013) 847-853. doi:10.1021/n14001053.

[4] S. Lee, W. Ko, Y. Oh, J. Lee, G. Baek, Y. Lee, J. Sohn, S. Cha, J. Kim, J. Park, J. Hong, Triboelectric energy harvester based on wearable textile platforms employing various surface morphologies, Nano Energy. 12 (2015) 410-418.

doi:10.1016/j.nanoen.2015.01.009.

[5] W. Seung, M.K. Gupta, K.Y. Lee, K. Shin, J. Lee, T.Y. Kim, S. Kim, J. Lin, J.H. Kim, S. Kim, Nanopatterned Textile-Based Wearable Triboelectric Nanogenerator, ACS Nano. 9 (2015) 3501-3509. doi:10.1021/nn507221f.

[6] X.S. Zhang, M. Di Han, R.X. Wang, B. Meng, F.Y. Zhu, X.M. Sun, W. Hu, W. Wang, Z.H. Li, H.X. Zhang, High-performance triboelectric nanogenerator with enhanced energy density based on single-step fluorocarbon plasma treatment, Nano Energy. 4 (2014) 123131. doi:10.1016/j.nanoen.2013.12.016.

[7] Y. Liu, J. Mo, Q. Fu, Y. Lu, N. Zhang, S. Wang, S. Nie, Enhancement of Triboelectric Charge Density by Chemical Functionalization, Adv. Funct. Mater. 30 (2020) 1-33. doi:10.1002/adfm.202004714.

[8] J. Luo, Z.L. Wang, Recent advances in triboelectric nanogenerator based self-charging power systems, Energy Storage Mater. (2019). doi:10.1016/j.ensm.2019.03.009.

[9] J. Luo, Z.L. Wang, Recent progress of triboelectric nanogenerators: From fundamental theory to practical applications, EcoMat. 2 (2020) 1-22. doi:10.1002/eom2.12059.

[10] Q. Gao, T. Cheng, Z.L. Wang, Triboelectric mechanical sensors-Progress and prospects, Extrem. Mech. Lett. 42 (2021) 101100. doi:10.1016/j.eml.2020.101100.

[11] W. Paosangthong, R. Torah, S. Beeby, Recent progress on textile-based triboelectric nanogenerators, Nano Energy. 55 (2019) 401-423. doi:10.1016/j.nanoen.2018.10.036.

[12] W. Wang, A. Yu, J. Zhai, Z.L. Wang, Recent Progress of Functional Fiber and Textile Triboelectric Nanogenerators: Towards Electricity Power Generation and Intelligent Sensing, Adv. Fiber Mater. (2021). doi:10.1007/s42765-021-00077-9.

[13] Y. Zou, V. Raveendran, J. Chen, Wearable triboelectric nanogenerators for biomechanical energy harvesting, Nano Energy. 77 (2020) 105303. doi:10.1016/j.nanoen.2020.105303.

[14] J. Xiong, P.S. Lee, Progress on wearable triboelectric nanogenerators in shapes of fiber, yarn, and textile, Sci. Technol. Adv. Mater. 20 (2019) 837-857.

doi:10.1080/14686996.2019.1650396.

[15] W. Wang, A. Yu, X. Liu, Y. Liu, Y. Zhang, Y. Zhu, Y. Lei, M. Jia, J. Zhai, Z.L. Wang, Large-scale fabrication of robust textile triboelectric nanogenerators, Nano Energy. 71 (2020) 104605. doi:10.1016/j.nanoen.2020.104605.

[16] S. Wang, Y. Xie, S. Niu, L. Lin, Z.L. Wang, Freestanding triboelectric-layer-based 
nanogenerators for harvesting energy from a moving object or human motion in contact and non-contact modes, Adv. Mater. 26 (2014) 2818-2824. doi:10.1002/adma.201305303.

[17] Y. Xie, S. Wang, S. Niu, L. Lin, Q. Jing, J. Yang, Z. Wu, Z.L. Wang, Grating-Structured Freestanding Triboelectric-Layer Nanogenerator for Harvesting Mechanical Energy at 85\% Total Conversion Efficiency, Adv. Mater. 26 (2014) 6599-6607. doi:10.1002/adma.201402428.

[18] L. Lin, S. Wang, Y. Xie, Q. Jing, S. Niu, Y. Hu, Z.L. Wang, Segmentally structured disk triboelectric nanogenerator for harvesting rotational mechanical energy, Nano Lett. 13 (2013) 2916-2923. doi:10.1021/nl4013002.

[19] P. Bai, G. Zhu, Y. Liu, J. Chen, Q. Jing, W. Yang, J. Ma, G. Zhang, Z.L. Wang, Cylindrical Rotating Triboelectric Nanogenerator, ACS Nano. 7 (2013) 6361-6366. doi:10.1021/nn402491y.

[20] H. Guo, Q. Leng, X. He, M. Wang, J. Chen, C. Hu, Y. Xi, A Triboelectric Generator Based on Checker-Like Interdigital Electrodes with a Sandwiched PET Thin Film for Harvesting Sliding Energy in All Directions, Adv. Energy Mater. 5 (2015) 1400790. doi:10.1002/aenm.201400790.

[21] X. Xia, G. Liu, H. Guo, Q. Leng, C. Hu, Y. Xi, Honeycomb-like three electrodes based triboelectric generator for harvesting energy in full space and as a self-powered vibration alertor, Nano Energy. 15 (2015) 766-775. doi:10.1016/j.nanoen.2015.05.033.

[22] G. Ma, B. Li, S. Niu, J. Zhang, D. Wang, Z. Wang, L. Zhou, Q. Liu, L. Liu, J. Wang, Z. Han, L. Ren, A bioinspired triboelectric nanogenerator for all state energy harvester and self-powered rotating monitor, Nano Energy. 91 (2022) 106637.

doi:10.1016/j.nanoen.2021.106637.

[23] J. Chen, H. Guo, X. Pu, X. Wang, Y. Xi, C. Hu, Traditional weaving craft for one-piece self-charging power textile for wearable electronics, Nano Energy. 50 (2018) 536-543. doi:10.1016/j.nanoen.2018.06.009.

[24] K. Dong, J. Deng, Y. Zi, Y.-C. Wang, C. Xu, H. Zou, W. Ding, Y. Dai, B. Gu, B. Sun, Z.L. Wang, 3D Orthogonal Woven Triboelectric Nanogenerator for Effective Biomechanical Energy Harvesting and as Self-Powered Active Motion Sensors, Adv. Mater. 29 (2017) 1702648. doi:10.1002/adma.201702648.

[25] Z. Tian, J. He, X. Chen, Z. Zhang, T. Wen, C. Zhai, J. Han, J. Mu, X. Hou, X. Chou, C. Xue, Performance-boosted triboelectric textile for harvesting human motion energy, Nano Energy. 39 (2017) 562-570. doi:10.1016/j.nanoen.2017.06.018.

[26] A. Yu, X. Pu, R. Wen, M. Liu, T. Zhou, K. Zhang, Y. Zhang, J. Zhai, W. Hu, Z.L. Wang, Core-Shell-Yarn-Based Triboelectric Nanogenerator Textiles as Power Cloths, ACS Nano. 11 (2017) 12764-12771. doi:10.1021/acsnano.7b07534.

[27] X. Hou, J. Zhu, J. Qian, X. Niu, J. He, J. Mu, W. Geng, C. Xue, X. Chou, Stretchable Triboelectric Textile Composed of Wavy Conductive-Cloth-PET and Patterned Stretchable Electrode for Harvesting Multi-variant Human Motion Energy, ACS Appl. Mater. Interfaces. 10 (2018) acsami.8b16267. doi:10.1021/acsami.8b16267. 
[28] W. Paosangthong, M. Wagih, R. Torah, S. Beeby, Textile-based triboelectric nanogenerator with alternating positive and negative freestanding grating structure, Nano Energy. 66 (2019) 104148. doi:10.1016/j.nanoen.2019.104148.

[29] F. Galembeck, T.A.L. Burgo, L.B.S. Balestrin, R.F. Gouveia, C.A. Silva, A. Galembeck, Friction, tribochemistry and triboelectricity: Recent progress and perspectives, RSC Adv. 4 (2014) 64280-64298. doi:10.1039/c4ra09604e.

[30] Z.L. Wang, A.C. Wang, On the origin of contact-electrification, Mater. Today. 30 (2019) 34-51. doi:10.1016/j.mattod.2019.05.016.

[31] A. Šutka, K. Malnieks, L. Lapčinskis, P. Kaufelde, A. Linarts, A. Berziṇa, R. Zabels, V. Jurķans, I. Gorņevs, J. Blums, M. Knite, The role of intermolecular forces in contact electrification on polymer surfaces and triboelectric nanogenerators, Energy Environ. Sci. 12 (2019) 2417-2421. doi:10.1039/c9ee01078e.

[32] S. Niu, Y. Liu, S. Wang, L. Lin, Y.S. Zhou, Y. Hu, Z.L. Wang, Theoretical Investigation and Structural Optimization of Single-Electrode Triboelectric Nanogenerators, Adv. Funct. Mater. 24 (2014) 3332-3340. doi:10.1002/adfm.201303799.

[33] Sheng Zhou, J.K. Pollard, Position Measurement using Bluetooth, IEEE Trans. Consum. Electron. 52 (2006) 555-558. doi:10.1109/TCE.2006.1649679.

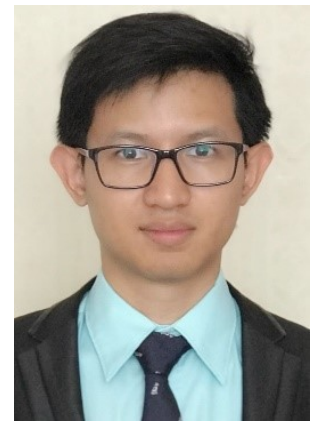

Watcharapong Paosangthong is a PhD student in Smart Electronic Materials and Systems Research Group at the University of Southampton, UK. He received his Diplom-Physiker Univ. degree in Physics from the Technische Universität München, Germany in 2011 and his MSc degree in MicroElectroMechanical Systems from the University of Southampton, UK, in 2017. From 2012 to 2016, he worked as research assistant and process engineer at Thai Microelectronics Center, Thailand. His research interests include triboelectric generator, energy harvesting, sensor systems, e-textiles and MEMS.

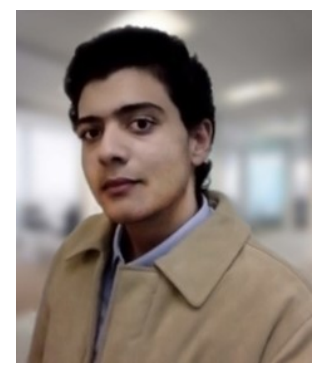

Mahmoud Wagih received his BEng in electrical and electronic engineering from the University of Southampton in 2018, where he received the best undergraduate project in sensors and instrumentation prize, 2018. He is currently pursuing his $\mathrm{PhD}$ at the University of Southampton. His current research interests lie in RF energy harvesting, wearable antennas, micro-power management and wireless sensor networks. He is a graduate student member of the IEEE, a reviewer for 2 IEEE Journals, and the recipient of the Best Student Paper Prize at the IEEE MTT-S WPTC conference, London, U.K. 2019. 


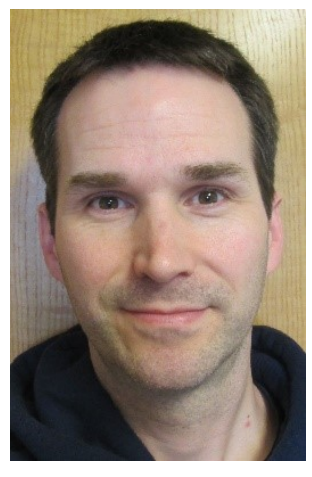

Russel Torah graduated with a BEng(hons) in Electronic Engineering and an MSc in Instrumentation and Transducers, both from the University of Southampton. In 2004 Russel obtained a $\mathrm{PhD}$ in Electronics from the University of Southampton. Since 2005 he has been a full-time researcher at the University of Southampton where he is currently a Principal Research Fellow. In 2011 Dr Torah co-founded Smart Fabric Inks Ltd specialising in printed smart fabrics. His research interests are currently focused on smart fabric development but he also has extensive knowledge of energy harvesting, sensors and transducers. Dr Torah has 150 publications and 2 patents.

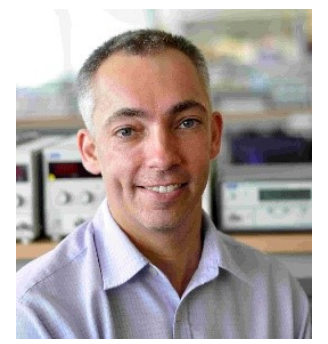

Professor Steve P Beeby received the B.Eng. (Hons.) degree in mechanical engineering from the University of Portsmouth, Portsmouth, U.K., in 1992, and the Ph.D. degree in MEMS resonant sensors from the University of Southampton, Southampton, U.K., in 1998. He is currently the Director of the Centre for Flexible Electronics and E-Textiles and leads the U.K.'s E-Textiles Network. He is leading three U.K. funded research projects and has received over $£ 16$ million research funding. He is a cofounder of Perpetuum Ltd., a University spin-out based upon vibration energy harvesting formed in 2004, Smart Fabric Inks Ltd., and D4 Technology Ltd. He has coauthored/edited five books including Energy Harvesting for Autonomous Systems (Artech House, 2010). He has given 29 invited talks and has over 300 publications and 9 patents with over 18,000 citations and an h-Index of 54. His current research interests focus on e-textiles and energy harvesting the use of energy harvesting in wearable applications. Prof. Beeby is the recipient of a prestigious RAEng Chair in Emerging Technologies and has previously received two EPSRC Research Fellowships to investigate the combination of screen-printed active materials with micromachined structures and textiles for energy harvesting. He was also awarded a Personal Chair in 2011. He is currently the Chair of the International Steering Committee for the PowerMEMS Conference series. 